MIT-CTP-3077

February 2001

\title{
The Spin Structure of the Nucleon: Theoretical Overview
}

\author{
Presented at the \\ Second Workshop on Physics with a Polarized-Electron Light-Ion Collider (EPIC) \\ September 14-16, 2000 at MIT, Cambridge, Massachusetts, USA \\ R.L. Jaffe \\ Center for Theoretical Physics and Department of Physics \\ Laboratory for Nuclear Physics Massachusetts Institute of Technology \\ Cambridge, Massachusetts 02139
}

\begin{abstract}
I review what is known about the quark and gluon spin distributions in the nucleon. I discuss in some detail (a) the existence of sum rules for angular momentum; (b) the interpretation and possible measurement of the nucleon's transversity distributions; and (c) the uses of spin-dependent fragmentation functions.
\end{abstract}

\section{INTRODUCTION}

The modern era in QCD spin physics dates from the 1987 discovery by the European Muon Collaboration that only about 30\% of the proton's spin is found on the spin of quarks [1]. Since then, particle and nuclear physicists have dreamt of facilities where QCD spin physics could be explored in detail. The recent commissioning of polarized $p p$ physics at RHIC is the first of these to be realized [2]. Our topic - a polarized ep collider in the energy regime where perturbative QCD meets confinement - is a necessary complement to $\overline{\mathrm{RHIC}}$ and the natural next step in unravelling the mysteries of quark confinement in QCD.

Among friends, I do not need to belabor the case for studying QCD at the boundary between the confining and perturbative domains. Two brief comments will suffice: First, quantum chromodynamics is the only nontrivial quantum field theory that we are certain describes the real world; and second, we need further experimental input to understand the highly complex QCD bound states that compose matter.

This workshop focuses on spin. While spin is an important degree of freedom, it is not the only important probe of confinement in deep inelastic processes. Flavor, twist, and quark mass dependence (through the substitution $u \rightarrow d \rightarrow s \rightarrow c \rightarrow b \rightarrow t$ ) yield different and complementary insights into the structure of QCD bound states. Spin, however, is today's topic. 
Of course it is impossible to cover the breadth of this field in a single talk. Fortunately, others will address important subjects in detail later at this meeting. Instead, I will make a brief survey of the present situation, emphasizing our present understanding of quark and gluon distribution functions, and then focus on three issues of current interest:

- Is there an "angular momentum sum rule" and is it experimentally testable?

- What is transversity and why is it interesting?

- Why are fragmentation functions interesting and useful in the study of spin in QCD?

\section{THE PRESENT SITUATION}

Polarization effects in QCD present a complex picture. Asymmetries need to be explained, but sometimes even if we cannot understand them, we can use them to probe other issues or isolate other important effects. Many striking asymmetries occur in the low energy or nuclear domain where we have few theoretical insights into QCD [3]. Most recent progress has occurred where the deep inelastic and soft domains overlap - the world of parton distribution and fragmentation functions. Here, spin effects help elucidate the puzzling nature of hadrons and here is where I will concentrate.

\section{A. Recent Events}

To set the stage for the workshop, here are lists of recent developments in experiment and theory, and a menu for expectations in the immediate future. First, experimental milestones of the past five years:

- First estimates of $\Delta g\left(x, Q^{2}\right)$ from evolution.

- First good look at $g_{2}\left(x, Q^{2}\right)$ from SLAC.

- First measurement of $\mu_{s} \equiv\left\langle\frac{1}{2} \vec{r} \times s^{\dagger} \vec{\alpha} s\right\rangle$ from SAMPLE.

- First fragmentation asymmetry measurements from Hermes.

- Commissioning of the polarized $p p$ component of RHIC.

Next, theory milestones of the past five years:

- Theory of $\Delta g$ measurements: Via evolution, via $\bar{c} c$ production, via $\vec{p}_{\|} \vec{p}_{\|} \rightarrow \gamma$ jet $X$.

- Development of the physics program for $\overrightarrow{\mathrm{RHIC}}$.

- Off-forward parton distributions and their possible measurement in deeply virtual Compton scattering (DVCS).

- The theory of the nucleon's angular momentum. 
- The theory of transversity and proposals to measure it.

- The classification of spin and transverse momentum effects in distribution and fragmentation processes.

And finally, prospects for the near future:

- Direct measurements of $\Delta g$.

- First measurements of transversity.

- First measurements of polarization-dependent fragmentation functions.

- Study of the inclusive/exclusive connection (i.e., higher twist), photoproduction, and DVCS at JLab.

- Flavor separation of the quark spin distributions.

- High quality measurements of $\Delta q\left(x, Q^{2}\right)$ at very low and very high $x$.

Clearly this field - the study of QCD confinement dynamics using polarized probes requires more than a single facility. Low $Q^{2}$ and low energy are needed for DVCS and for studies of higher twist. High $Q^{2}$ is needed to study $\Delta g$ via evolution. High energy is necessary to create the phase space for complex final states such as $\bar{c} c$ studies of $\Delta g$ and multijet final states. Both polarized lepton beams and polarized proton beams are required. High-density polarized targets are required for high-luminosity studies of $g_{2}$ at SLAC and extraction of neutron distributions from polarized deuterium and ${ }^{3} \mathrm{He}$ scattering data.

While we enthuse about the particular subject of this workshop, we must remember that the field requires an opportunistic, even predatory mentality, ready to make use of many facilities in imaginative ways.

\section{B. Bjorken's Sum Rule}

Occasionally it is worth reminding ourselves what it means to "understand" something in QCD. In the absence of fundamental understanding we often invoke "effective descriptions" based on symmetries and low-energy expansions. While they can be extremely useful, we should not forget that a thorough understanding allows us to relate phenomena at very different distance scales to one another. In the case case of Bjorken's sum rule, the operator product expansion, renormalization group invariance and isospin conservation combine to relate deep inelastic scattering at high $Q^{2}$ to the neutron's $\beta$-decay axial charge measured at very low energy. Even target mass and higher twist corrections are relatively well understood. The present state of the sum rule is

$$
\begin{aligned}
\int_{0}^{1} \mathrm{~d} x g_{1}^{e p-e n}\left(x, Q^{2}\right)=\frac{1}{6} \frac{g_{A}}{g_{V}} & \left\{1-\frac{\alpha_{s}\left(Q^{2}\right)}{\pi}-\frac{43}{12} \frac{\alpha_{s}^{2}\left(Q^{2}\right)}{\pi^{2}}-20.215 \frac{\alpha_{s}^{3}\left(Q^{2}\right)}{\pi^{3}}\right\} \\
+ & \frac{M^{2}}{Q^{2}} \int_{0}^{1} x^{2} \mathrm{~d} x\left\{\frac{2}{9} g_{1}^{e p-e n}\left(x, Q^{2}\right)+\frac{1}{6} g_{2}^{e p-e n}\left(x, Q^{2}\right)\right\} \\
& -\frac{1}{Q^{2}} \frac{4}{27} \mathcal{F}^{u-d}\left(Q^{2}\right)
\end{aligned}
$$


where the three lines correspond to QCD [4], target mass, and higher twist [5] corrections respectively; $g_{1}$ and $g_{2}$ are the nucleon's longitudinal and transverse spin-dependent structure functions; $g_{A}$ and $g_{V}$ are the neutron's $\beta$-decay axial and vector charges. $\mathcal{F}$ is a twist-4 operator matrix element with dimensions of $[\text { mass }]^{2}$, which measures a quark-gluon correlation within the nucleon,

$$
\mathcal{F}^{u}\left(Q^{2}\right) s^{\alpha}=\frac{1}{2}\left\langle P S\left|g \bar{u} \widetilde{F}^{\alpha \lambda} \gamma_{\lambda} u\right|_{Q^{2}} \mid P S\right\rangle
$$

where $g$ is the QCD coupling, $\widetilde{F}$ is the dual gluon field strength, and $\left.\right|_{Q^{2}}$ denotes the operator renormalization point.

The most thorough analysis of the Bj sum rule I know of is one presented by SMC in 1998 [6]. Their theoretical evaluation gives

$$
\left.\int_{0}^{1} \mathrm{~d} x g_{1}^{e p-e n}\left(x, Q^{2}\right)\right|_{\text {theory }}=0.181 \pm 0.003
$$

at $Q^{2}=5 \mathrm{GeV}^{2}$. Experiment is not yet able to reach this level of accuracy. The latest data relevant to the Bj sum rule is shown in Fig. 1. The value extracted by the $\mathrm{SMC}$ is

$$
\left.\int_{0}^{1} \mathrm{~d} x g_{1}^{e p-e n}\left(x, Q^{2}\right)\right|_{\text {expt }}=0.174 \pm 0.05 \begin{array}{rr}
+0.011 & +0.021 \\
-0.009 & -0.006
\end{array}
$$

at $Q^{2}=5 \mathrm{GeV}^{2}$, and the errors are statistical, systematic, and "theoretical" (e.g., generated

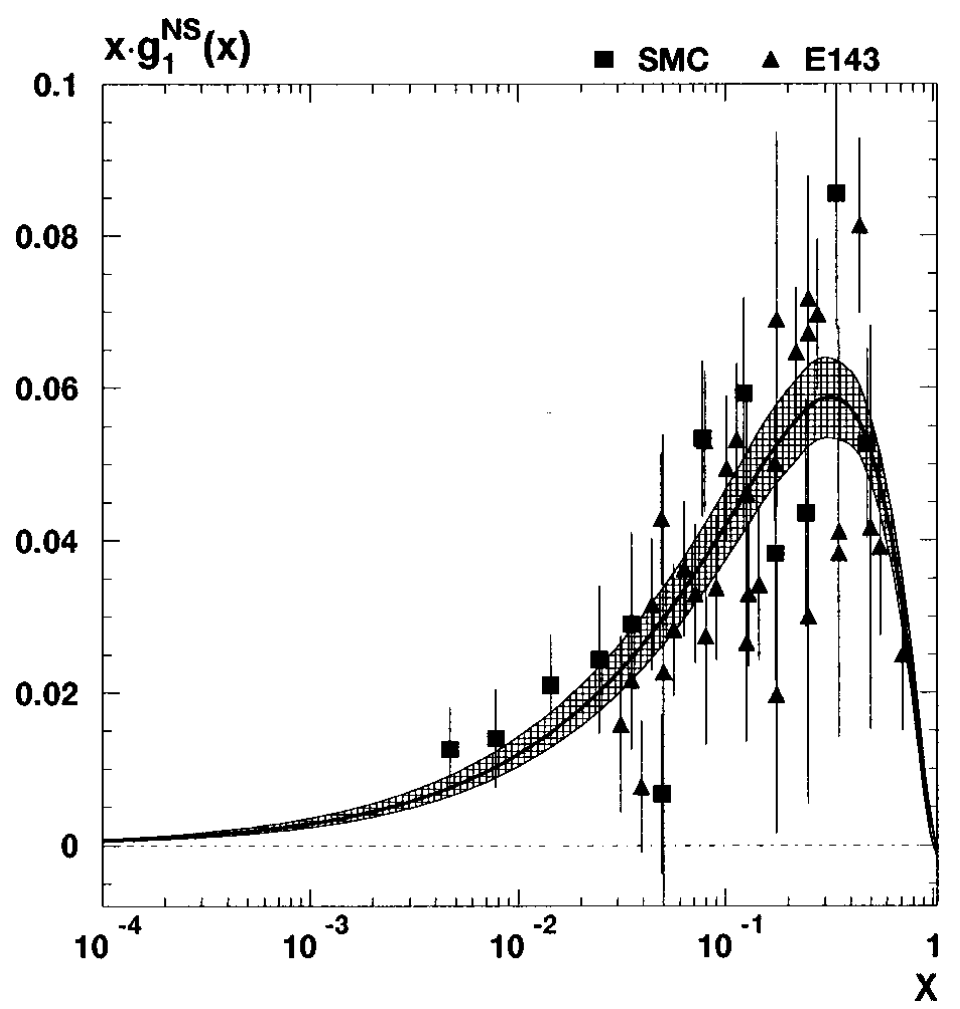

FIG. 1. SMC analysis of data relevant to the Bjorken sum rule.

by running the data to a common $Q^{2}$ ), respectively [6]. Further accuracy is necessary to confirm the target mass corrections and extract the twist-four contribution. 


\section{Quark and gluon distributions in the nucleon}

No overview of the nucleon's spin structure is complete without a survey of the polarized quark and gluon distributions in the nucleon. These helicity-weighted momentum distributions are the most precise and interpretable information we have about the spin substructure of a hadron. The distributions are usually defined in terms of flavor-SU(3) structure,

$$
\begin{aligned}
\text { Singlet: } & \Delta \Sigma=\Delta U+\Delta D+\Delta S \\
\text { Nonsinglet, isovector: } & \Delta q_{3}=\Delta U-\Delta D \\
\text { Nonsinglet, hypercharge: } & \Delta q_{8}=\Delta U+\Delta D-2 \Delta S
\end{aligned}
$$

where $\Delta Q \equiv q^{\uparrow}\left(x, Q^{2}\right)+\bar{q}^{\uparrow}\left(x, Q^{2}\right)-q^{\downarrow}\left(x, Q^{2}\right)-\bar{q}^{\downarrow}\left(x, Q^{2}\right)$. Experimenters seem to prefer nonsinglet distributions specialized to the proton and neutron individually,

$$
\begin{aligned}
\text { Proton nonsinglet: } & \Delta q_{N S}(p)=\Delta U-\frac{1}{2} \Delta D-\frac{1}{2} \Delta S \\
\text { Neutron nonsinglet: } & \Delta q_{N S}(n)=\Delta D-\frac{1}{2} \Delta U-\frac{1}{2} \Delta S
\end{aligned}
$$

so that

$$
\begin{aligned}
& g_{1}^{p}=\frac{2}{9} \Delta \Sigma+\frac{2}{9} \Delta q_{N S}(p) \\
& g_{1}^{n}=\frac{2}{9} \Delta \Sigma+\frac{2}{9} \Delta q_{N S}(n) .
\end{aligned}
$$

Since the integrated quark spin accounts for only about $30 \%$ of the nucleon's spin, it is extremely interesting to know whether the integrated gluon spin in the nucleon is large. Of course the polarized gluon distribution, $\Delta g\left(x, Q^{2}\right)$, cannot be measured directly in deep inelastic scattering because gluons do not couple to the electromagnetic current. Instead, $\Delta g$ is inferred from the QCD evolution of the quark distributions. (See Ref. [6] for details of the process and references to the original literature.) However, evolution of imprecise data only constrains a few low moments of $\Delta g$ and gives only crude information on global characteristics such as the existence and number of nodes. It is clear that $\Delta g$ must be measured directly elsewhere.

That said, the world's data on polarized structure functions is summarized in Figs. 2 and 3. Fig. 2 is taken from Naomi Makins's talk at DIS2000 and presents the world's data on $g_{1}^{p}$ in the same format traditionally used for unpolarized structure function data [7]. The figure highlights the tremendous progress of the past decade as well as the need for much better data if our knowledge of polarized distributions would aspire to the same accuracy as unpolarized distributions. Note, in particular, that the entire kinematic domain over which $g_{1}$ has been measured would fit into the lower left-hand corner of the $F_{2}$ figure. Fig. 3 shows the quark and gluon distributions extracted from the world's data by SMC, together with estimates of systematic and theoretical uncertainties [6]. While the information on quark distributions is fairly precise, it is clear that we know very little about the distribution of polarized gluons in the nucleon. 

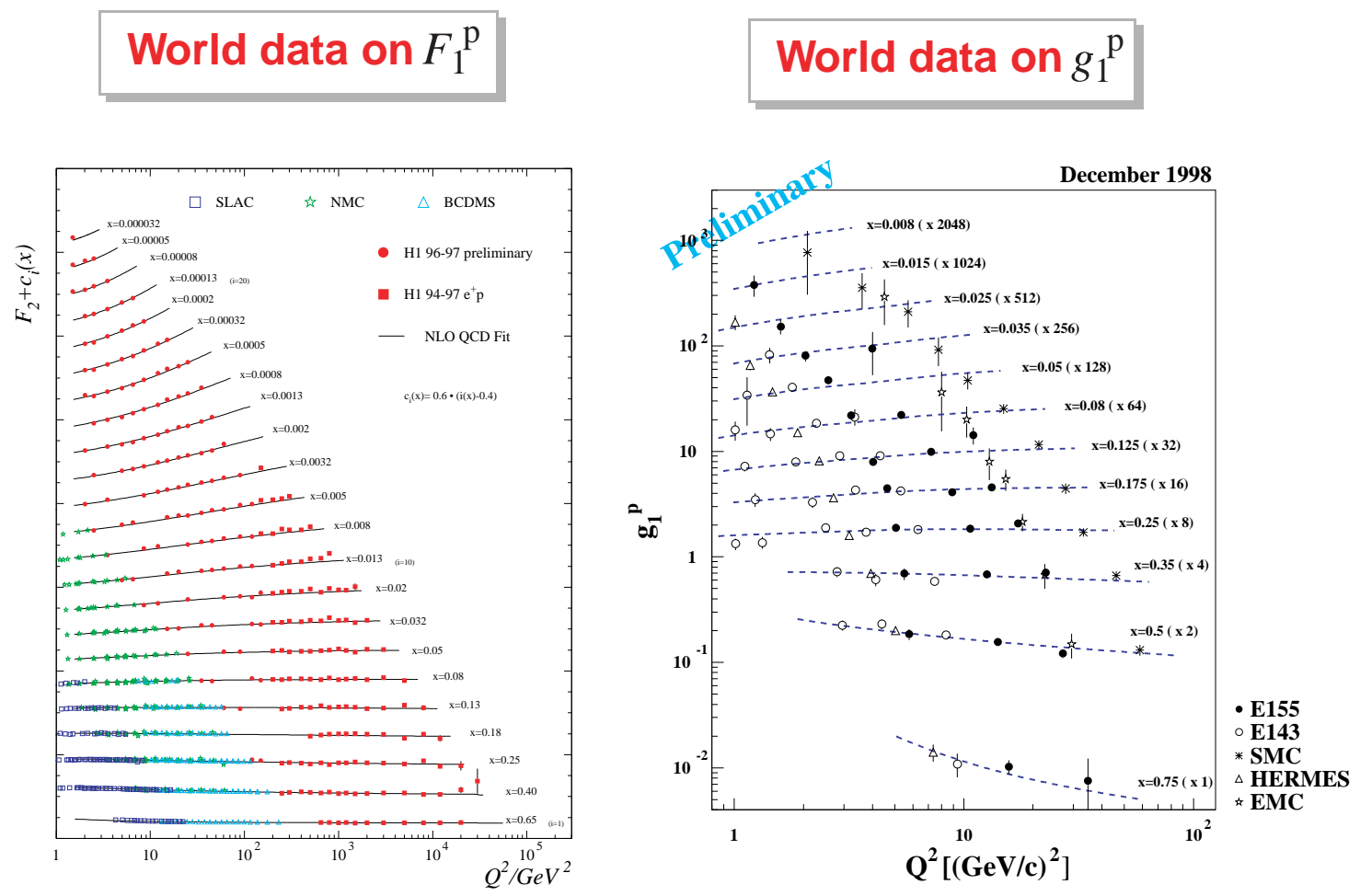

FIG. 2. World data on spin-average and spin-dependent structure functions [7].

\section{IS THERE AN "ANGULAR MOMENTUM SUM RULE" AND IS IT EXPERIMENTALLY TESTABLE?}

It has been clear for years that in some sense the nucleon's spin (projected along an axis) can be written as a sum of contributions from quark and gluon spin and orbital angular momentum [9],

$$
\frac{1}{2}=\frac{1}{2} \Delta \Sigma+\Delta g+L_{q}+L_{g}
$$

but the interpretation and usefulness of such a relation has only recently been clarified. The principal issues are

- Are the terms separately gauge-invariant?

- Are they interaction-dependent?

- Is each separately measurable?

- Is each related to an integral over a parton $x$-distribution?

I believe we can now answer these questions, but the answers are not what we would like.

I want to distinguish between two different kinds of relations with the form of Eq. 8. A "classic" sum rule expresses the expectation value of a local operator in a state as an integral 

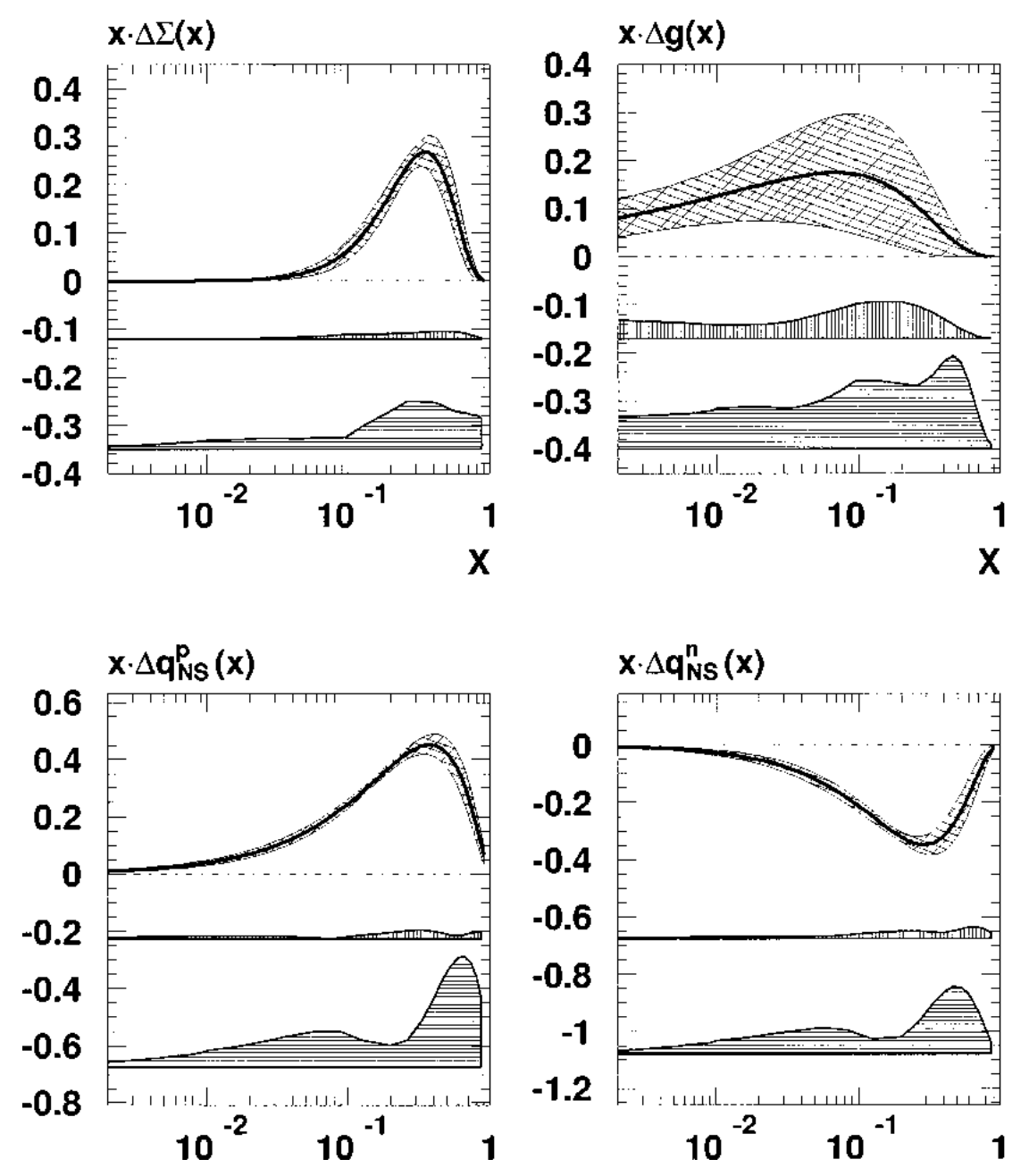

FIG. 3. Polarized quark and gluon distribution functions. The upper figures show the distribution with a statistical error bound. The lower figures show estimates of systematic and theoretical uncertainties, respectively.

(or sum) over a distribution measured in an inelastic production process involving the same state. This is the traditional definition of a "sum rule", dating back to the Thomas-ReicheKuhn sum rule of atomic spectroscopy. All the familiar sum rules of deep inelastic scattering - Bjorken's, Gross \& Llewellyn-Smith's, etc. - are this type of relation. They are even more powerful because the distribution that is integrated has a simple, heuristic interpretation as the momentum (Bjorken- $x$ ) distribution of the observable associated with the local operator. The "spin sum rule" gives a typical example:

$$
\begin{aligned}
\left\langle P, S\left|\bar{q}_{a} \gamma^{\mu} \gamma_{5} q_{a}\right|_{Q^{2}} \mid P, S\right\rangle / S^{\mu} & \equiv \Delta q_{a}\left(Q^{2}\right) \\
& =\int_{0}^{1} \mathrm{~d} x\left\{q_{a \uparrow}\left(x, Q^{2}\right)+\bar{q}_{a \uparrow}\left(x, Q^{2}\right)-q_{a \downarrow}\left(x, Q^{2}\right)-\bar{q}_{a \downarrow}\left(x, Q^{2}\right)\right\}
\end{aligned}
$$

The left-hand side can be measured in $\beta$-decay or other electroweak processes. The righthand side can be measured in deep inelastic scattering of polarized leptons from polarized targets. The meaning of the sum rule is clear because the local operator, $\bar{q}_{a} \gamma^{\mu} \gamma_{5} q_{a}$, is the 
generator of the internal rotations (the "spin") of the quark field in QCD. The sum rule says that the quark's contribution to the nucleon's spin is the integral over a spin-weighted momentum distribution of the quarks.

Another, less powerful, but still interesting type of relation - sometimes called a sum rule - arises simply because an operator can be written as the sum of two (or more) other operators, $\Theta=\Theta_{1}+\Theta_{2}$. If the expectation values of all three operators can be measured, then this relation, and the assumptions underlying it, can be tested. Such a relation exists for the contributions to the nucleon's angular momentum [9]:10],

$$
\frac{1}{2}=\hat{L}_{q}+\frac{1}{2} \Sigma+\hat{J}_{g}
$$

where the three terms are roughly the quark orbital angular momentum, the quark spin, and the total angular momentum on the gluons. Ji has shown how, in principle, to measure the various terms in this relation [10].

A sum rule of the classic type also exists for the contributions to the nucleon's angular momentum, 13, 12, 14]

$$
\frac{1}{2}=\int_{0}^{1} \mathrm{~d} x\left\{L_{q}\left(x, Q^{2}\right)+\frac{1}{2} \Delta q\left(x, Q^{2}\right)+L_{g}\left(x, Q^{2}\right)+\Delta g\left(x, Q^{2}\right)\right\}
$$

where the four terms are precisely the $x$-distributions of the quark orbital angular momentum, quark spin, gluon orbital angular momentum, and gluon spin. However, it appears that the distributions $L_{q}\left(x, Q^{2}\right)$ and $L_{g}\left(x, Q^{2}\right)$ are not experimentally accessible. So the value of the sum rule is obscure.

Before exploring these relations for the angular momentum in more depth, let's examine the simpler and well-understood case of energy and momentum.

\section{A. Sum rules for energy and momentum}

One hears a lot about the "momentum sum rule" in QCD, but nothing about an "energy sum rule". The reasons are quite instructive. Energy and momentum are described by the rank-two, symmetric energy-momentum tensor, $T^{\mu \nu}$,

$$
T^{\mu \nu}=\frac{i}{4} \bar{q}\left(\gamma^{\mu} D^{\nu}+\gamma^{\nu} D^{\mu}\right) q+\text { h.c. }+\operatorname{Tr}\left(F^{\mu \alpha} F_{\alpha}^{\nu}-\frac{1}{4} g^{\mu \nu} F^{2}\right)
$$

where $D^{\mu}$ and $F^{\mu \nu}$ are the gauge covariant derivative and gluon field strength, both matrices in the fundamental representation of $\mathrm{SU}(3)$. [ $T^{\mu \nu}$ is ambiguous up to certain total derivatives, but these do not change the arguments presented here.]

The energy density is given by $T^{00}$,

$$
\mathcal{E} \equiv T^{00}=\frac{1}{2} q^{\dagger}(-i \vec{\alpha} \cdot \vec{D}+\beta m) q+\text { h.c. }+\operatorname{Tr}\left(\vec{E}^{2}+\vec{B}^{2}\right) .
$$

The expectation value of $T^{00}$ is normalized,

$$
\left\langle P\left|T^{00}\right| P\right\rangle=2 E^{2},
$$


because $|P\rangle$ is an eigenstate of the Hamiltonian, $\int \mathrm{d}^{3} x T^{00}(x)|P\rangle=E|P\rangle$. This is a good start towards a sum rule. However there is no useful sum rule because there is no way to write any of the terms in Eq. (13) as an integral over inelastic production data. This is not obvious, but the appearance of terms in $\mathcal{E}$ that are order cubic and higher in the canonical fields is a bad sign. The first term in $\mathcal{E}$ includes $\bar{q} q g$ coupling, and $\vec{E}^{2}+\vec{B}^{2}$ involves terms cubic and quartic in the gluon vector potentials $\vec{A}$. The parton distributions of deep inelastic scattering (DIS) come from operators quadratic in the "good" light-cone components of the quark and gluon fields, $q_{+}$and $\vec{A}_{\perp}$ [11].

In contrast there is a classic, deep-inelastic sum rule for $P^{+}$, where $P^{+}=\frac{1}{\sqrt{2}}\left(P^{0}+P^{3}\right)$, and the 3 -direction is singled out by the gauge choice $A^{+}=0 . T^{++}$is normalized much like $T^{00}$,

$$
\left\langle P\left|T^{++}\right| P\right\rangle=2 P^{+2}
$$

Unlike $T^{00}, T^{++}$simplifies dramatically in $A^{+}=0$ gauge because of the simplification of $D^{+}$and $F^{+\alpha}$,

$$
\begin{aligned}
D^{+} & =\partial^{+}-i g A^{+} \rightarrow \partial^{+} \\
F^{+\alpha} & =\partial^{+} A^{\alpha}-\partial^{\alpha} A^{+}+g\left[A^{+}, A^{\alpha}\right] \rightarrow \partial^{+} A^{\alpha} .
\end{aligned}
$$

As a result $T^{++}$is quadratic in the fundamental dynamical variables, $q_{+}$and $\vec{A}_{\perp}$ and all interactions disappear,

$$
T^{++}=i q_{+}^{\dagger} \partial^{+} q_{+}+\operatorname{Tr}\left(\partial^{+} \vec{A}_{\perp}\right)^{2} .
$$

The two terms give the contributions of quarks and gluons respectively to the total $P^{+}$. It is straightforward to relate each to an integral over a positive definite parton "momentum" distribution,

$$
\begin{aligned}
i q_{+}^{\dagger} \partial^{+} q & \rightarrow \int \mathrm{d} x x q(x) \\
\left(\partial^{+} \vec{A}_{\perp}\right)^{2} & \rightarrow \int \mathrm{d} x x g(x)
\end{aligned}
$$

in which the parton probability density is weighted by the observable (in this case $x$ ) appropriate to the sum rule. Keeping track of renormalization scale dependence and kinematic factors of $P^{+}$, one obtains the standard "Momentum" sum rule,

$$
1=\int_{0}^{1} \mathrm{~d} x x\left\{q\left(x, Q^{2}\right)+g\left(x, Q^{2}\right)\right\}
$$

The lessons learned from this exercise generalize to the more difficult case of angular momentum:

- The time components of the tensor densities associated with space-time symmetries do not yield classic sum rules. Interactions do not drop out. They yield relations that are difficult to interpret because quark and gluon contributions do not separate. Individual terms are not related to integrals over parton distributions.

- The +-components of the same tensor densities do yield useful sum rules, which have a parton interpretation in $A^{+}=0$ gauge. Interactions drop out. Each term can be represented as an integral over a parton distribution weighted by the appropriate observable quantity. 


\section{B. Sum rules for angular momentum}

The situation for angular momentum is not satisfactory. The time-component analysis yields a relation, some of whose ingredients can be measured (in principle) in deeply virtual Compton scattering. But it has no place for a separately gauge-invariant gluon spin and orbital angular momentum, no clean separation between quark and gluon contributions, and no relation to quark or gluon $x$ distributions. The +-component analysis yields a classic sum rule with separate quark and gluon spin and orbital angular momentum contributions, each gauge invariant, each related to a parton distribution, and each free from interaction terms. Unfortunately, there does not seem to be a way to measure the terms in this otherwise perfectly satisfactory sum rule.

The tensor density associated with rotations and boosts is a three component tensor antisymmetric in the last two indices, $M^{\mu \nu \lambda}$. To extract a sum rule, we polarize the nucleon along the 3-direction in its rest frame and set $\nu=1, \lambda=2$ in order to select rotations about this direction. The matrix elements of $M^{012}$ and $M^{+12}$ are both normalized in terms of the nucleon's momentum $\left(P^{\mu}=(M, 0,0,0)\right)$ and spin $\left(S^{\mu}=(0,0,0, M)\right)$ [9].

First consider the time component, $M^{012}$,

$$
M^{012}=\frac{i}{2} q^{\dagger}(\vec{x} \times \vec{D})^{3} q+\frac{1}{2} q^{\dagger} \sigma^{3} q+2 \operatorname{Tr} E^{j}(\vec{x} \times \vec{D})^{3} A^{j}+\operatorname{Tr}(\vec{E} \times \vec{A})^{3} .
$$

The four terms look like the generators of rotations (about the 3-axis) for quark orbital, quark spin, gluon orbital, and gluon spin angular momentum respectively. Taking the matrix element in a nucleon state at rest one obtains,

$$
\frac{1}{2}=\hat{L}_{q}+\frac{1}{2} \Sigma+\hat{L}_{g}+\Delta \hat{g}
$$

There are problems, however. There are no parton representations for $\hat{L}_{g}, \hat{L}_{q}$, or $\Delta \hat{g}$, so it is not a sum rule in the classic sense. $\Sigma$ is the integral of the helicity weighted quark distribution, but $\Delta \hat{g}$ is not the integral of the helicity weighted gluon distribution. Interactions prevent a clean separation into quark and gluon contributions as they did for $T^{00}$. And worse still, $\hat{L}_{g}$ and $\Delta \hat{g}$ are not separately gauge invariant, so only the sum $\hat{J}_{g}=\hat{L}_{g}+\Delta \hat{g}$ is physically meaningful.

The most important feature of the relation, Eq. (21), is the result derived by Ji, that

$$
\hat{J}_{q}=\hat{L}_{q}+\frac{1}{2} \Sigma
$$

and $\hat{J}_{g}$ can, in principle, be measured in deeply virtual Compton scattering [10]. In practice, $\hat{L}_{q}$ may be measurable, but $\hat{J}_{g}$ can only be obtained by $Q^{2}$ evolution of $\hat{J}_{q}$, which seems beyond experimental attack for the foreseeable future. Without a handle on $\hat{J}_{g}$ and given the ambiguity in the definition of $\hat{L}_{q}$ (see below), the usefulness of Eq. 22 is unclear.

Turning to the +-component sum rule, we find a much simpler form,

$$
M^{+12}=\frac{1}{2} q_{+}^{\dagger}(\vec{x} \times \vec{i} \partial)^{3} q_{+}+\frac{1}{2} q_{+}^{\dagger} \gamma_{5} q_{+}+2 \operatorname{Tr} F^{+j}(\vec{x} \times i \vec{\partial}) A^{j}+\operatorname{Tr} \epsilon^{+-i j} F^{+i} A^{j}
$$

in $A^{+}=0$ gauge. [This gauge condition must be supplemented by the additional condition that the gauge fields vanish fast enough at infinity.] The four terms in $M^{+12}$ correspond 
respectively to quark orbital angular momentum, quark spin, gluon orbital angular momentum, and gluon spin, all about the 3 -axis. Each is separately gauge invariant ${ }^{\text {mand involves }}$ only the "good", i.e., dynamically independent, degrees of freedom, $q_{+}$and $\vec{A}_{\perp}$. Each is a generator of the appropriate symmetry transformation in light-front field theory. The resulting sum rule,

$$
\frac{1}{2}=L_{q}+\frac{1}{2} \Sigma+L_{g}+\Delta g
$$

is a classic deep inelastic sum rule. It can be written as an integral over $x$-distributions

$$
\frac{1}{2}=\int_{0}^{1} \mathrm{~d} x\left\{L_{q}\left(x, Q^{2}\right)+\frac{1}{2} \delta q\left(x, Q^{2}\right)+L_{g}\left(x, Q^{2}\right)+\Delta g\left(x, Q^{2}\right)\right\}
$$

where each term is an interaction independent, gauge invariant, integral over a partonic density associated with the appropriate symmetry generator [12 14].

Satisfying though eqs. (24) and (25) may be from a theoretical point of view. They are quite useless unless someone finds a way to measure the two new terms $L_{q}$ and $L_{g}$.

\section{TRANSVERSITY}

One of the major accomplishments of the recent renaissance in QCD spin physics has been the rediscovery and exploration of the quark transversity distribution. First mentioned by Ralston and Soper in 1979 in their treatment of Drell-Yan $\mu$-pair production by transversely polarized protons [15], the transversity was not recognized as a major component in the description of the nucleon's spin until the early 1990s [16 18, 11].

The transversity can be interpreted in parton language as follows: consider a nucleon moving with (infinite) momentum in the $\hat{e}_{3}$-direction, but polarized along one of the directions transverse to $\hat{e}_{3} . \delta q_{a}\left(x, Q^{2}\right)$ counts the quarks of flavor $a$ and momentum fraction $x$ with their spin parallel the spin of a nucleon minus the number antiparallel. If quarks moved nonrelativistically in the nucleon, $\delta q$ and $\Delta q$ would be identical, since rotations and Euclidean boosts commute and a series of boosts and rotations can convert a longitudinally polarized nucleon into a transversely polarized nucleon at infinite momentum. So the difference between the transversity and helicity distributions reflects the relativistic character of quark motion in the nucleon. There are other important differences between transversity and helicity. For example, quark and gluon helicity distributions $(\Delta q$ and $\Delta g)$ mix under $Q^{2}$-evolution. There is no analog of gluon transversity in the nucleon, so $\delta q$ evolves without mixing, like a nonsinglet distribution function. The lowest moment of the transversity is proportional to the nucleon matrix element of the tensor charge, $\bar{q} i \sigma^{0 i} \gamma_{5} q$, which couples only to valence quarks (it is $C$-odd). Not coupling to glue or $\bar{q} q$ pairs, the tensor charge

\footnotetext{
${ }^{1}$ Note, however, that in any gauge other than $A^{+}=0$, the operators are nonlocal and appear to be interaction dependent. The same happens to the simple operators involved in the momentum sum rule, Eq. (17).
} 
promises to be more quark-model-like than the axial charge and should be an interesting contrast.

We now know that the transversity, $\delta q\left(x, Q^{2}\right)$, together with the unpolarized distribution, $q\left(x, Q^{2}\right)$, and the helicity distribution, $\Delta q\left(x, Q^{2}\right)$, are required to give a complete description of the quark spin in the nucleon at leading twist. An equation tells this story clearly:

$\mathcal{A}\left(x, Q^{2}\right)=\frac{1}{2} q\left(x, Q^{2}\right) I \otimes I+\frac{1}{2} \Delta q\left(x, Q^{2}\right) \sigma_{3} \otimes \sigma_{3}+\frac{1}{2} \delta q\left(x, Q^{2}\right) \quad\left(\sigma_{+} \otimes \sigma_{-}+\sigma_{-} \otimes \sigma_{+}\right)$.

Here, $\mathcal{A}$ is the quark distribution in a nucleon as a density matrix in both the quark and nucleon helicities (hence the external product of two Pauli matrices in each term), diagrammatically equivalent to the lower part of the handbag diagram shown in Fig. Ha; $q$ governs

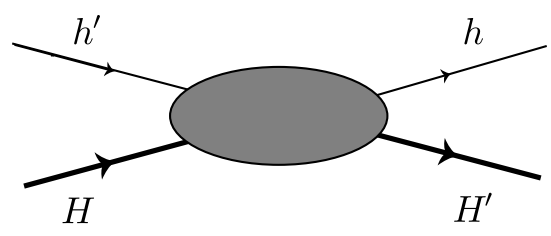

(a)

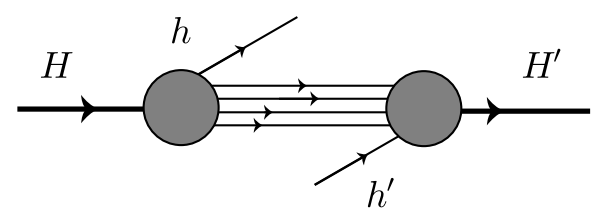

(b)

FIG. 4. Quark hadron forward scattering. Quark helicities are labeled $h$ and $h^{\prime}$; hadron helicities are $H$ and $H^{\prime}$. (a) Full scattering amplitude; (b) u-channel discontinuity, which gives the quark distribution function in DIS.

spin average physics, $\Delta q$ governs helicity dependence, and $\delta q$ governs helicity flip - or transverse polarization - physics.

In terms of the helicity amplitude $\mathcal{A}_{H h, H^{\prime} h^{\prime}}$ in Fig. $4 \mathrm{~b} \mathrm{~b}$, the transversity is given by $\mathcal{A}_{++,--}$, corresponding to quark and nucleon helicity flip. The spin average $(q)$ and helicity $(\Delta q)$ distributions involve $\mathcal{A}_{++,++}, \mathcal{A}_{+-,+-}$, which preserve quark helicity. The connection between transverse spin and helicity flip is a consequence of simple quantum mechanics. The two states of transverse polarization can be written as superpositions of helicity eigenstates: $\left|\frac{\perp}{\top}\right\rangle=\frac{1}{\sqrt{2}}(|+\rangle \pm|-\rangle)$; the cross section with transverse polarization has the form $d \sigma_{\perp} \propto\left\langle\stackrel{\perp}{T}_{T}|\ldots| \frac{\perp}{\top}\right\rangle$; so the difference of cross sections is proportional to helicity flip, $d \sigma_{\perp}-d \sigma_{\top} \propto\langle+|\ldots|-\rangle+\langle-|\ldots|+\rangle$. At leading twist, quark helicity and chirality are identical. For this reason, the transversity distribution are called "chiral-odd", in contrast to the "chiral-even" distributions, $q$ and $\Delta q$.

Quark chirality is conserved at all QCD and electroweak vertices; however, quark chirality can flip in distribution and fragmentation functions because they probe the soft regime where chiral symmetry is dynamically broken in QCD. This is another reason to be interested in 
transversity - it probes dynamical chiral symmetry breaking, an incompletely understood aspect of QCD.

Because all hard QCD and electroweak processes preserve chirality, transversity is difficult to measure. It decouples from inclusive DIS and most other familiar deep inelastic processes. The argument is made graphically in Fig. 5. In order to access transversity

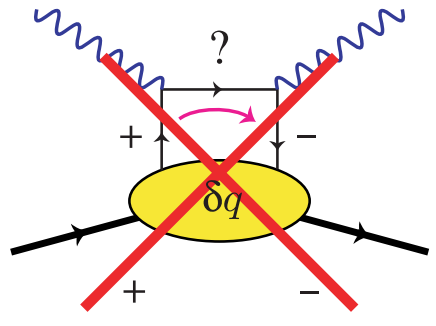

(a)

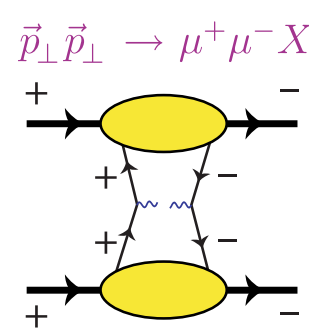

(b)

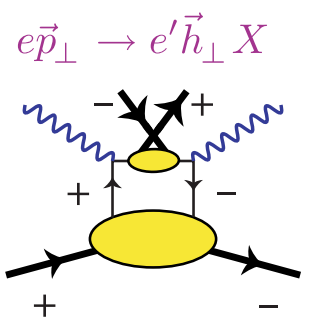

(c)

FIG. 5. Deep inelastic processes relevant to transversity.

some second soft process must flip the quark chirality a second time. The classic example, where transversity was discovered by Ralston and Soper, is transversely polarized Drell-Yan production of muon pairs: $\vec{p}_{\perp} \vec{p}_{\perp} \rightarrow \mu^{+} \mu^{-} X$, which is shown diagrammatically in Fig. 5(b). Chirality is flipped in both soft distribution functions and the cross section is proportional to $\delta q\left(x_{1}, Q^{2}\right) \times \delta \bar{q}\left(x_{2}, Q^{2}\right)$.

Transversity would not decouple from deep inelastic scattering if some electroweak vertex would flip chirality. Unfortunately (and accidentally from the point of view of QCD) all photon, $W^{ \pm}$and $Z^{0}$ couplings all preserve chirality. Quark-Higgs couplings violate chirality but are too weak to be of interest. Quark mass insertions flip chirality, and indeed a careful analysis reveals effects proportional to $m \delta q\left(x, Q^{2}\right) / \sqrt{Q^{2}}$ in inclusive DIS with a transversely polarized target. However the $u, d$, and $s$ quarks, which are common in the nucleon, are too light to give significant sensitivity to $\delta q$.

What is needed is an insertion that flips chirality without introducing a $1 / \sqrt{Q^{2}}$ suppression. A generic example is shown in Fig. 5(c). Much interest has been generated recently by the observation of an asymmetry at Hermes that can be interpreted as evidence for a chirality-flipping fragmentation function that couples to the nucleon's transversity. It corresponds to a particular instance of Fig. 5(c). If this effect is confirmed it suggests a bright future for transversity measurements at the next generation of polarized lepton-hadron facilities.

\section{FRAGMENTATION AND SPIN: THE HERMES ASYMMETRY AND BEYOND}

To my mind, the single most interesting development in QCD spin physics over the past two years is the azimuthal asymmetry in pion electroproduction reported by Hermes [19]. It is interesting both in itself and as an emblem of a new class of spin measurements involving spin-dependent fragmentation processes, which act as filters for exotic parton distribution functions like transversity. 


\section{A. Fragmentation functions as probes of unstable hadrons}

Fragmentation functions allow us to access and explore the spin structure of unstable hadrons, which cannot be used as targets for deep inelastic scattering. These include the $\rho$, $\omega$, and $\phi$ mesons, and hyperons like the $\Lambda$ and $\Sigma$. Let me give three examples:

\section{The tensor fragmentation function of the $\rho$}

When a quark of helicity $h= \pm \frac{1}{2}$ fragments collinearity into a $\rho$ of helicity $H=1$, 0 , or -1 , there are many fragmentation functions, $\mathcal{F}_{H h, H^{\prime} h^{\prime}}$, in analogy to $\mathcal{A}_{H h, H^{\prime} h^{\prime}}$ discussed in the previous section. If we consider fragmentation of helicity eigenstates, then the fragmentation functions can be labelled by the quark helicity $h$ and the $\rho$ helicity $H$ corresponding to $q_{h} \rightarrow \rho_{H}$. Parity relates three pairs, e.g., $q_{\frac{1}{2}} \rightarrow \rho_{1}=q_{-\frac{1}{2}} \rightarrow \rho_{-1}$, leaving three independent combinations. These can be classified as the spin average: $q \rightarrow \rho$; the helicity difference:

$\left(q_{\frac{1}{2}} \rightarrow \rho_{1}\right)-\left(q_{\frac{1}{2}} \rightarrow \rho_{-1}\right)$; and the tensor fragmentation function, known as $\hat{b}_{\rho}$ in analogy to the tensor distribution function first analyzed in connections with the deuteron [20]: $\hat{b}_{\rho}=\left(q \rightarrow \rho_{1}\right)+\left(q \rightarrow \rho_{-1}\right)-2\left(q \rightarrow \rho_{0}\right)$. The function $\hat{b}_{\rho}$ is independent of quark spin and has the simple physical interpretation of measuring the difference between quark fragmentation into a transverse $\rho$ compared to a longitudinal $\rho$. The $\pi \pi$ angular distribution in $\rho$ decay is sensitive to $\hat{b}_{\rho}$, so it can be measured [21]. The data are already available. The challenge to theorists is to make use of it.

\section{The $\rho$ double-helicity flip-fragmentation function}

Consider the fragmentation of a gluon into a $\rho$. In addition to the fragmentation functions already discussed for quarks, a double helicity flip fragmentation function can occur. The process and the helicity labels are shown in Fig. 6. This process has a unique signature in the $\pi \pi$ angular distribution and no equivalent in $q \rightarrow \rho$. So it is a special probe of gluon fragmentation into the $\rho$.

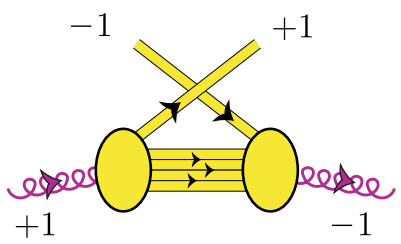

FIG. 6. Figure showing gluon and rho helicity labels in the double helicity flip case.

\section{Polarized quark $\rightarrow$ polarized $\Lambda$ fragmentation}

It should be clear that one can define longitudinal and transverse spin dependent fragmentation functions of the $\Lambda$, schematically $\vec{q}_{\|} \rightarrow \vec{\Lambda}_{\|}$and $\vec{q}_{\perp} \rightarrow \vec{\Lambda}_{\perp}$, in direct analogy to the quark helicity and transversity distributions in a target $\Lambda$. Since the $\Lambda \rightarrow p \pi$ decay is 
self-analyzing, it is relatively easy to measure the spin of the $\Lambda$. By selecting $\Lambda$ 's produced in the current fragmentation region one can hope to isolate the fragmentation process $q \rightarrow \Lambda$. Having measured the quark spin structure of the nucleon, we can use flavor-SU(3) to estimate the way quark spins are distributed in the $\Lambda$ [22]. However we do not know if this information is reflected in the fragmentation process $q \rightarrow \Lambda$. Once again the challenge is to theorists to learn how to interpret fragmentation functions in a heuristic way analogous to the quark parton model of distribution functions.

\section{B. Fragmentation as a filter for novel distribution functions}

Even if we do not know how to interpret fragmentation functions, we can use them as filters, to select parton distribution functions that either decouple from or are hard to extract from completely inclusive DIS. The simplest and best known example is the use of meson flavor to tag strange versus nonstrange quark distribution functions. This analysis has been developed to a high level of sophistication by the Hermes collaboration who use the felicitous term "purity" to denote the propensity for strange quarks to fragment to strange mesons and so forth [24]. They identify "favored" fragmentation processes like $u \rightarrow \pi^{+}$and "disfavored" processes like $u \rightarrow \pi^{-}$and set up a transfer matrix formalism to give a complete characterization of $e N \rightarrow e^{\prime}(\pi, K, \eta) X$. The interest is not principally in the various fragmentation functions, but instead to use them as filters for specific quark (and antiquark) distribution functions. Hermes and Compass hope to use these methods to extract the polarized antiquark distributions in the nucleon, $\Delta \bar{u}\left(x, Q^{2}\right), \Delta \bar{d}\left(x, Q^{2}\right)$, and $\Delta \bar{s}\left(x, Q^{2}\right)$. Their competition in this pursuit comes from $\overrightarrow{\mathrm{RHIC}}$, where $W^{ \pm}$production asymmetries can be used to trigger on specific quark flavors and extract $\Delta \bar{u}$ and $\Delta \bar{d}$.

A more complex, and potentially much more interesting example is the use of a helicity flip fragmentation function to select the quark transversity distribution. As shown in Fig. 5(c), by interposing a helicity flip fragmentation function on the struck quark line in DIS, it is possible to access the transversity. What is needed is a twist-two, chiral-odd fragmentation function. There are several candidates:

- $\delta \hat{q}_{a}\left(z, Q^{2}\right)$, the transverse, spin-dependent fragmentation function. This is the analog in fragmentation of transversity, and describes the fragmentation of a transversely polarized quark into a transversely polarized hadron with momentum fraction $z$ [25,26]. To access $\delta \hat{q}$, it is necessary to measure the spin of a particle in the final state of DIS. In practice this limits the application to production of a $\Lambda$ hyperon - the only particle whose spin is easy to measure through its parity violating decay.

- $\delta \hat{q}_{I}\left(z, m^{2}, Q^{2}\right)$, the two pion interference fragmentation function. 27 29 This describes the fragmentation of a transversely polarized quark into a pair of pions whose orbital angular momentum is correlated with the quark spin. This requires measurement of two pions in the final state. It may be quite useful, especially in polarized collider experiments [30]. I will not discuss it further here.

- $\hat{c}\left(z, Q^{2}\right)$, the single particle azimuthal asymmetry fragmentation function. This function, first discussed by Collins, et al. [27], describes the azimuthal distribution of pions about the axis defined by the struck quark's momentum in deep inelastic scattering. 
All three of these fragmentation functions are chiral-odd and therefore produce experimental signatures sensitive to the transversity distribution in the target nucleon. Each may play an important role in future experiments aimed at probing the nucleon's transversity. Recently Hermes has announced observation of a spin asymmetry that seems to be associated with the Collins function, $c\left(z, Q^{2}\right)$. So although all three deserve discussion, I will spend the rest of my time on the Collins function and the Hermes asymmetry.

\section{The Collins Fragmentation Function}

The standard description of fragmentation without polarization requires a single fragmentation function usually called $D_{h}(z)$. It gives the probability that a quark will fragment into a hadron, $h$, with longitudinal momentum fraction $z$. [For simplicity I suppress the dependence of $D$ on the virtuality scale, $Q^{2}$ and the quark flavor label $a$.] The transverse momentum of $h$ relative to the quark is integrated out. If the transverse momentum, $\vec{p}_{\perp}$, is observed, then it is possible to construct distributions weighted by geometric factors. For instance,

where, for comparison,

$$
c(z) \propto \int \mathrm{d}^{2} p_{\perp} D_{h}\left(z, \vec{p}_{\perp}\right) \cos \chi
$$

$$
D(z) \propto \int \mathrm{d}^{2} p_{\perp} D_{h}\left(z, \vec{p}_{\perp}\right) .
$$

Here $D_{h}\left(z, \vec{p}_{\perp}\right)$ is the probability for the quark to fragment into hadron $h$ with momentum fraction $z$ and transverse momentum $\vec{p}_{\perp} ; \chi$ is the angle between $\vec{p}_{\perp}$ and some vector, $\vec{w}$, defined by the initial state. Since we don't know the direction of the quark's momentum exactly, the transverse momentum of the hadron, $\vec{p}_{\perp}$, is defined relative to some large, externally determined momentum, such as the momentum of the virtual photon, $\vec{q}$, in DIS.

How can $c(z)$ figure in deep inelastic scattering? The trick is to find a vector, $\vec{w}$, relative to which $\chi$ can be defined. If the target is polarized, it is possible to define $\vec{w}$ by taking the cross product of the target spin, $\vec{s}$, with either the initial or final electron's momentum $\left(\vec{k}\right.$ or $\vec{k}^{\prime}$ ) depending on the circumstances. Generically, then, the observable associated with $c(z)$ is $\cos \chi \propto \vec{k} \times \vec{s} \cdot \vec{p}$, where $\vec{p}$ is the momentum of the observed hadron in the final state. The situation is illustrated in Fig. (7) from Ref. [31]. This observable is even under

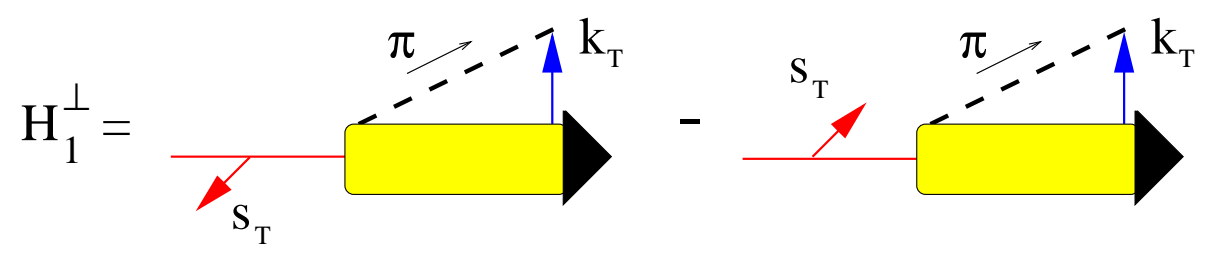

FIG. 7. The Collins effect function $H_{1}^{\perp}$ signals different probabilities for $q(\perp / \perp) \rightarrow \pi\left(\vec{k}_{\perp}\right)+X$.

parity (because $\vec{s}$ is a pseudovector), but odd under time reversal. This does not mean that it violates time-reversal invariance. Instead it means that it will vanish unless there are final state interactions capable of generating a nontrivial phase in the DIS amplitude. This 
subtlety makes it hard to find a good model to estimate $c(z)$ because typical fragmentation models involve only tree graphs (if they involve quantum mechanics at all!), which are real.

The Collins fragmentation function, $c(z)$, may be interesting in itself, but it is much more interesting because it is chiral-odd and combines with the transversity distribution in the initial nucleon to produce an experimentally observable asymmetry sensitive to the transversity. Two specific cases figure in recent and soon-to-be-performed experiments.

\section{Single particle inclusive DIS with a transversely polarized target: $e \vec{p}_{\perp} \rightarrow e^{\prime} \pi X$}

If the target is transversely polarized (with respect to the initial electron momentum, $\vec{k}$ ), then $\vec{w}=\vec{k} \times \vec{s}$ defines a vector normal to the plane defined by the beam and the target spin. The transverse momentum of the produced hadron can be defined either with respect to the beam or the momentum transfer $\vec{q}$ - the difference in higher order in $1 / Q \cdot \cos \chi$ is defined by $\cos \chi=\vec{p}_{\perp} \cdot \vec{w} /\left|\vec{p}_{\perp}\right||\vec{w}|$. The kinematics are particularly simple in this case (transverse spin). Experimenters prefer to think of the effect in terms of the angle $(\phi)$ between two planes: Plane 1 is defined by the virtual photon and the target spin, and Plane 2 is defined by the virtual photon and the transverse momentum of the produced hadron. Then $\sin \phi=\cos \chi$ and the effect is known as a "sin $\phi$ " asymmetry. When the cross section is weighted by $\sin \phi$, the result is

$$
\frac{\mathrm{d} \Delta \sigma_{\perp}}{\mathrm{d} x \mathrm{~d} y \mathrm{~d} z}=\frac{2 \alpha^{2}}{Q^{2}} \sum_{a} e_{a}^{2} \delta q_{a}(x) c_{a}(z)
$$

where $y=E-E^{\prime} / E$, and $\Delta \sigma$ is the difference of cross sections with target spin reversed. $L^{2}$ This is a leading twist effect, which scales (modulo logarithms of $Q^{2}$ ) in the deep inelastic limit. If $c(z)$ is not too small, it is will become the "classic" way to measure the nucleon's transversity distributions.

No experimental group has yet measured hadron production in deep inelastic scattering from a transversely polarized target, so there is no data on $\Delta \sigma_{\perp}$. Hermes at DESY intend to take data under these conditions in the next run. One reason for this was the observation of a $\sin \phi$ asymmetry with a longitudinally polarized target that Hermes announced last year [19]. It strongly suggests, but does not require, that $\Delta \sigma_{\perp}$ should be large.

\section{Single particle inclusive DIS with a longitudinally polarized target: $e \vec{p}_{\|} \rightarrow e^{\prime} \pi X$}

The possibility of a $\sin \phi$ asymmetry is more subtle in this case, and it escaped theorists' attention for a long time. The possibility of such an asymmetry was first pointed out in Ref [32]. As $Q^{2}$ and $\nu$ go to $\infty$, the initial and final electrons' momenta become parallel. If the target spin is parallel to $\vec{k}$, then it is impossible to construct a vector from $\vec{k}$ or $\vec{k}^{\prime}$

\footnotetext{
${ }^{2}$ In principle, this reversal is superfluous because the $\sin \phi$ asymmetry must be odd under reversal and the rest of the cross section must be even. However, it helps experimenters to reduce systematic errors.
} 
and $\vec{s}$ in this limit. However, $\vec{k}$ and $\vec{k}^{\prime}$ are not exactly parallel, so $\vec{s}$ has a small component perpendicular to the virtual photon's momentum, $\vec{q}=\vec{k}-\vec{k}^{\prime}$. The vector, $\vec{w}$, can be defined as $\vec{w}=\vec{k}^{\prime} \times \vec{s}$, and the kinematic situation is shown in Fig. 8 from Ref. [19]. This produces

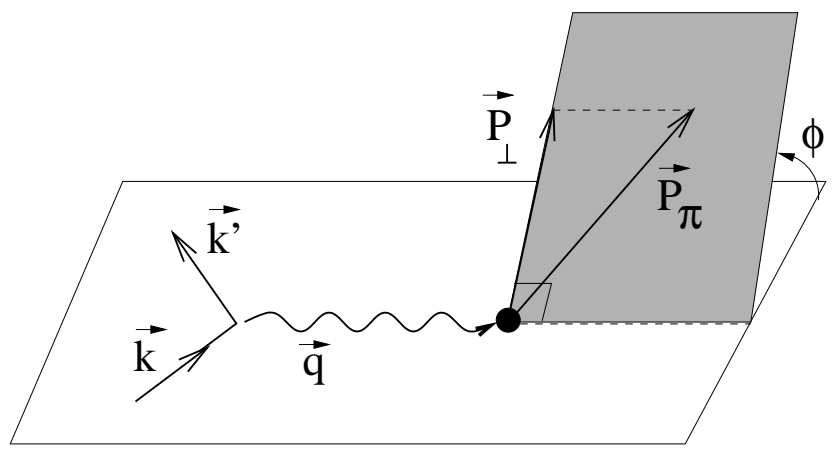

FIG. 8. Kinematic planes for pion production in semi-inclusive deep-inelastic scattering.

an asymmetry similar to the previous case, but weighted by $\left|\vec{s}_{\perp}\right| \propto 2 M x / Q$. Because this leading (twist two) effect is kinematically suppressed by $1 / Q$, it is necessary to consider other, twist-three, effects that might be competitive. A careful analysis turns up a variety of twist-three effects, leading to a cross section of the form [31, 32],

$$
\frac{\mathrm{d} \Delta \sigma_{\|}}{\mathrm{d} x \mathrm{~d} y \mathrm{~d} z}=\frac{2 \alpha^{2}}{Q^{2}} \frac{2 M x}{Q} \sqrt{1-y} \sum_{a} e_{a}^{2}\left\{\delta q_{a}(x) c_{a}(z)+\frac{2-y}{1-y} h_{L a}(x) c_{a}(z)\right\}
$$

where $h_{L}(x)$ is a longitudinal spin-dependent, twist-three distribution function analogous to $g_{T}$.

By far the most interesting thing about $\Delta \sigma_{\|}$is that Hermes has seen such an asymmetry in their $\pi^{+}$data. (The Hermes data is shown in Fig. 9.) They see no effect in their $\pi^{-}$data. Because $u$ quarks predominate in the nucleon, because $e_{u}^{2}=4 e_{d}^{2}$, and because $u \rightarrow \pi^{+} \ll u \rightarrow \pi^{-}$, they expect no signal in $\pi^{-}$. They have not reported on $\pi^{0}$, where an asymmetry similar to $\pi^{+}$would be expected.

If the Hermes result is confirmed, it demonstrates that the Collins fragmentation function is nonzero. Somehow the final state interactions between the observed pion and the other fragments of the nucleon suffice to generate a phase that survives the sum over the other unobserved hadrons. Whatever its origin, a nonvanishing Collins function would be a great gift to the community interested in the transverse spin structure of the nucleon. It provides an unanticipated tool for extracting the nucleon's transversity from DIS experiments. The fact that Hermes has seen a robust (2-3\%) asymmetry with a longitudinally polarized target suggests that they will see a large asymmetry with a transversely polarized target (unless the effect is entirely twist three - e.g., $h_{L} \gg \delta q$ ). This in turn will lead to the first measurements of the nucleon's transversity distribution and to new insight into the relativistic spin structure of confined states of quarks and gluons. 

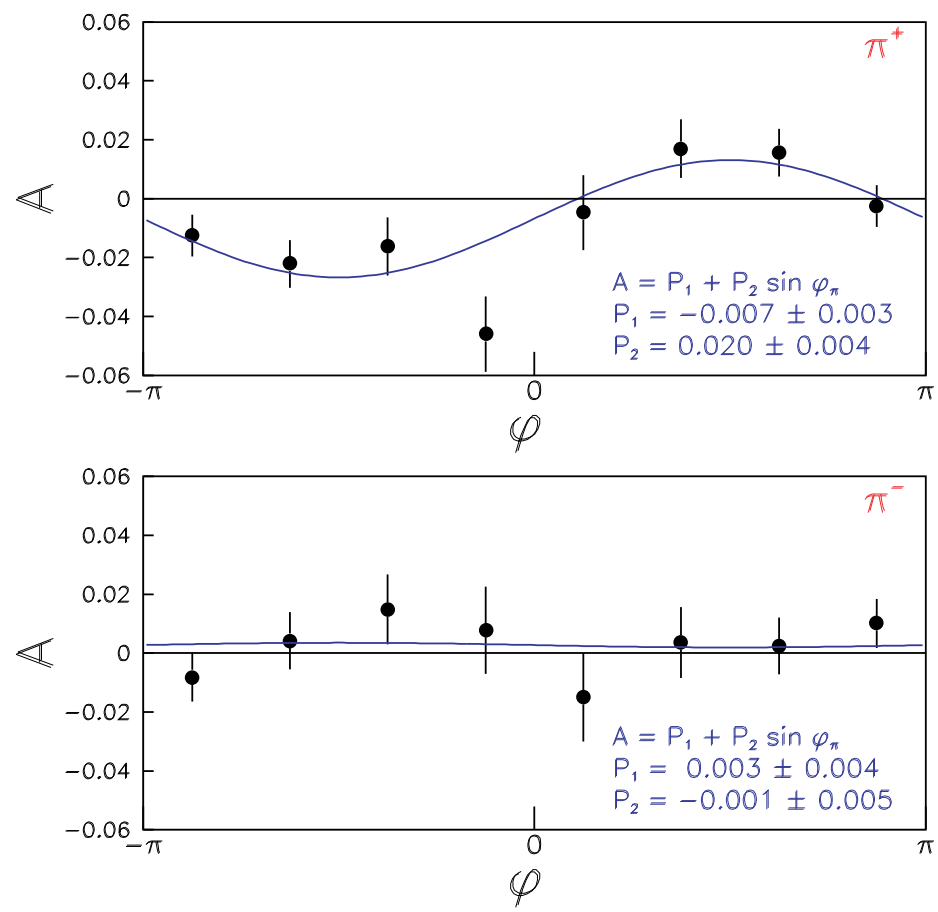

FIG. 9. Azimuthal asymmetry $(\sin \phi)$ distribution for $\pi+$ and $\pi^{-}$production with a longitudinally polarized target at Hermes.

\section{CONCLUSIONS}

A richer and more complex picture of the QCD bound states has emerged since the 1987 renaissance precipitated by the EMC observation that quarks carry only a small fraction of the nucleon spin. We know much more about the nucleon's spin than we did back then. We also know what to look for in the future: we have a clear program for future measurement and analysis of the gluon helicity distribution, $\Delta g$, the quark transversity, $\delta q_{a}$, and the flavor decomposition of the quark spin $(\Delta \bar{u}, \Delta \bar{d}$, etc.) and a host of other related subjects, which I have not had time to discuss here. This program involves several facilities and different energy regimes. The polarized ep collider we are considering at this workshop clearly has a central role to play.

\section{Acknowledgments}

This work is supported in part by funds provided by the U.S. Department of Energy (D.O.E.) under cooperative research agreement \#DF-FC02-94ER40818. 


\section{REFERENCES}

[1] J. Ashman et al. [European Muon Collaboration], Phys. Lett. B206, 364 (1988).

[2] N. Saito, "Spin Physics at RHIC", presented at the 14th International Spin Symposium, SPIN2000, Osaka, November 2000.

[3] A. D. Krisch, in SPIN 98 Proceedings of the13th International Symposium on High Energy Spin Physics Protvino, Russia 8 - 12 September 1998, N. E. Tyurin, V. L. Solovianov, S. M. Troshin, and A. G. Ufimtsev, eds. (World Scientific, Singapore, 1999)

[4] S. A. Larin and J. A. Vermaseren, Phys. Lett. B259, 345 (1991).

[5] E. V. Shuryak and A. I. Vainshtein, Nucl. Phys. B201, 141 (1982).

[6] B. Adeva et al. [Spin Muon Collaboration], Phys. Rev. D 58, 112002 (1998).

[7] N. C. R. Makins [for the Hermes Collaboration] Talk presented at DIS2000. To be published in the proceedings.

[8] G. Bunce, N. Saito, J. Soffer, and W. Vogelsang, hep-ph/0007218.

[9] R. L. Jaffe and A. Manohar, Nucl. Phys. B337, 509 (1990).

[10] X. Ji, Phys. Rev. Lett. 78, 610 (1997) hep-ph/9603249].

[11] R. L. Jaffe, hep-ph/9602236.

[12] P. Hagler and A. Schafer, Phys. Lett. B430, 179 (1998) hep-ph/9802362.

[13] A. Harindranath and R. Kundu, Phys. Rev. D59, 116013 (1999) hep-ph/9802406.

[14] S. V. Bashinsky and R. L. Jaffe, Nucl. Phys. B536, 303 (1998) hep-ph/9804397.

[15] J. P. Ralston and D. E. Soper, Nucl. Phys. B152, 109 (1979).

[16] X. Artru and M. Mekhfi, Z. Phys. C45, 669 (1990).

[17] R. L. Jaffe and X. Ji, Phys. Rev. Lett. 67, 552 (1991).

[18] J. L. Cortes, B. Pire, and J. P. Ralston, Z. Phys. C55, 409 (1992).

[19] A. Airapetian et al. [Hermes Collaboration], Phys. Rev. Lett. 84, 4047 (2000) hepex/9910062.

[20] P. Hoodbhoy, R. L. Jaffe, and A. Manohar, Nucl. Phys. B312, 571 (1989).

[21] A. Schafer, L. Szymanowski, and O. V. Teryaev, Phys. Lett. B464, 94 (1999) hepph/9906471].

[22] M. Burkardt and R. L. Jaffe, Phys. Rev. Lett. 70, 2537 (1993) hep-ph/9302232.

[23] R. L. Jaffe, Phys. Rev. D 54, 6581 (1996) hep-ph/9605456].

[24] J. M. Niczyporuk and E. E. Bruins, Phys. Rev. D 58, 091501 (1998) hep-ph/9804323.

[25] R. L. Jaffe and X. Ji, Phys. Rev. Lett. 71, 2547 (1993) hep-ph/9307329.

[26] D. Boer, hep-ph/0007047.

[27] J. C. Collins, S. F. Heppelmann, and G. A. Ladinsky, Nucl. Phys. B420, 565 (1994) hep-ph/9305309.

[28] J. C. Collins and G. A. Ladinsky, hep-ph/9411444.

[29] R. L. Jaffe, X. Jin, and J. Tang, Phys. Rev. Lett. 80, 1166 (1998) hep-ph/9709322.

[30] M. Grosse Perdekamp "Transversity Measurement at RHIC" presented at the 14th International Spin Symposium, SPIN2000, Osaka, November 2000.

[31] D. Boer, RIKEN Rev. 28, 26 (2000) hep-ph/9912311.

[32] K. A. Oganessyan, H. R. Avakian, N. Bianchi, and A. M. Kotzinian, hep-ph/9808368. 